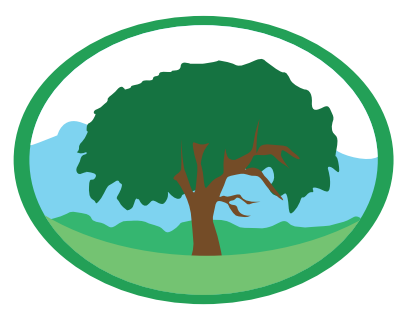

\title{
SUSCETIBILIDADE À INUNDAC̣ÃO DA BACIA HIDROGRÁFICA DO RIO PIRATINI - RS
}

MOURA, M. M. ${ }^{1}$, ARAÚJO, M. M. F. ${ }^{2}$, CASTRO, T. A. D. ${ }^{1}$, LEANDRO D. ${ }^{1}$

'Universidade Federal de Pelotas

2Universidade Federal do Rio Grande

Palavras-chave: classificação

Processos

Hierárquica,

Remoto.

\section{prevenção, Resumo}

de imagens, de Análise Sensoriamento

Nos últimos anos é perceptível o crescimento dos estudos acerca da suscetibilidade à inundação em bacias hidrográficas. Diversas técnicas vem sendo desenvolvidas para identificação de áreas suscetíveis, com destaque para os métodos multicritérios, os quais tem como finalidade minimizar falhas e auxiliar no processo de tomada de decisão. Este estudo tem como objetivo analisar a suscetibilidade da bacia hidrográfica do rio Piratini (BHRP), localizada no Estado do Rio Grande do Sul. Foi utilizado o método Analytic Hierarchy Process e os atributos analisados foram: declividade, altitude, classe de solo e uso do solo. Dois cenários foram propostos: i) solo exposto e ii) solo com cobertura vegetal. Pode-se perceber que: i) a suscetibilidade na BHRP teve variação similar nos dois cenários propostos; ii) a bacia apresentou maior porcentagem de áreas com baixa e muito baixa suscetibilidade durante o período com cobertura vegetal; iii) a porcentagem da bacia enquadrada com suscetibilidade muito alta duplicou no período com o solo exposto; e iv) as áreas próximas às zonas urbanas apresentam suscetibilidade alta ou muito alta durante todo o ano.

\section{SUSCEPTIBILITY TO FLOODING THE PIRATINI RIVER BASIN - RS}

Keywords: prevention, image classification, Analytic Hierarchy Process, Remote Sensing.

\section{Abstract}

In recent years, studies on the susceptibility to flooding in watersheds have grown. Several techniques have been developed to identify susceptible areas, especially multicriteria methods, which aim to minimize failures and assist in the decision making process. The objective of this study was to analyze the susceptibility of the Piratini river basin, located in the State of Rio Grande do Sul. The Analytic Hierarchy Process was used, and the attributes analyzed were: slope, altitude, soil class and ground. Two scenarios were proposed: i) soil exposed and ii) soil with vegetal cover. It can be noticed that: i) the susceptibility in the BHRP had similar variation in the two proposed scenarios; ii) the basin presented a higher percentage of areas with low and very low susceptibility during the period with vegetal cover; iii) the percentage of the basin framed with very high susceptibility doubled in the period with the soil exposed; and (iv) areas close to urban areas are subject to high or very high susceptibility throughout the year. 


\section{INTRODUÇ̃̃o}

Nos últimos anos é perceptível o crescimento dos estudos acerca da suscetibilidade à inundação de uma dada área. De acordo com Saavedra, Muñoz e Reis (2015), a suscetibilidade à inundação de uma região pode ser compreendida como o conjunto de condicionantes naturais que predispóem a mesma a ser inundada, e que podem ser observadas anteriormente à ocorrência de um evento. As análises apontam dois principais fatores que interferem na ocorrência desse fenômeno: fatores socioambientais e climáticos (YOUSSEF; PRADHAN; HASSAN, 2011).

Assim, variados autores debruçam-se sobre a literatura para embasar suas investigaçóes visando o desenvolvimento de novas metodologias para a identificação de áreas suscetíveis à inundação como, por exemplo, classificação de imagens obtidas após a ocorrência de eventos extremos (LIM; LEE, 2017; ZHANG; ZHU; LIU, 2014), caracterização morfométricas de bacias hidrográficas (SAHARIA et al., 2017; VIECELI et al., 2015) e identificação de áreas suscetíveis com base no modelo digital de elevação (MDE) (MCKENZIE; RUTTER; HULBERT, 2010; OLIVEIRA et al. 2018). Entretanto, em grande maioria destas metodologias, as condicionantes não são analisadas de forma conjunta, o que dificulta um entendimento mais completo da espacializaçáo do fenômeno ao longo da bacia hidrográfica.

Frente a estas limitaçóes, tem se destacado o método multicritério Analytic Hierarchy Process (AHP), proposto por Saaty (1977), que tem como finalidade minimizar falhas e auxiliar no processo de tomadas de decisooes (MARCHEZETTI; KAVISKI; BRAGA, 2011). Este método é amplamente utilizado, com destaque para estudos de suscetibilidade a escorregamentos (YALCIN et al., 2011; SHAHABI et al., 2014), análises de adequação de uso da terra (AK1NC1; ÖZALP; TURGUT, 2014; FEIZIZADEH; BLASCHKE, 2013) e suscetibilidade à inundação (ANDRADE; FERREIRA; SILVA, 2014; MAGALHÁES et al., 2011). De forma geral, observa-se que os parâmetros adotados para análise de suscetibilidade à inundação são: relevo, mapa de solos e mapa de uso do solo.

Diante do exposto, o objetivo do presente estudo é realizar uma análise da variabilidade espacial da suscetibilidade à inundação na bacia hidrográfica do rio Piratini, no sul do Brasil, tomando como base dois cenários: o primeiro com parte do solo descoberto, e o segundo, um período com cobertura vegetal.

\section{MATERIAL E MÉTODOS}

\section{Área de estudo}

O estudo compreende a bacia hidrográfica do rio Piratini (BHRP), localizada no Estado do Rio Grande do Sul (RS) (Figura 1A). A BHRP possui $4700,47 \mathrm{~km}^{2}$ e é um dos principais afluentes do Canal São Gonçalo, importante via navegável que une a Laguna dos Patos à Lagoa Mirim (GOUVÊA; ZARNOT; ALBA, 2010). Para este estudo, a BHRP foi delimitada tomando como exutório a estação de monitoramento fluviométrico Pedro Osório (88641000), sob responsabilidade da Agência Nacional de Águas (ANA) (Figura 1B).

De acordo com Álvares et al. (2013), adotando a classificação de Köppen, o clima da região é do tipo Cfa, subtropical úmido, sem estação seca, caracterizado por verôes quentes, chuvas bem distribuídas ao longo do ano e temperatura média do mês mais quente superior a $22^{\circ} \mathrm{C}$ e do mês mais frio variando entre $-3^{\circ} \mathrm{C}$ e $18^{\circ} \mathrm{C}$.

\section{Base de dados}

O software de geoprocessamento ArcGIS $10.1^{\oplus}$ (ESRI, 2014) foi utilizado para o processamento da base de dados e álgebra de mapas. Foram utilizadas quatro imagens da Shuttle Radar Topography Mission (SRTM), com resolução espacial de $30 \mathrm{~m}$, para compor o MDE de toda a região de estudo. As imagens s32_w053, s32_w054, s33_w053 e s33_w054 foram obtidas no banco de dados do United States Geological Survey (USGS) e mosaicadas.

Com base no MDE, a BHRP foi delimitada e as altitudes foram extraídas para o recorte da bacia hidrográfica, a qual foi delimitada por meio da ferramenta Extract by Mask e, posteriormente, foram classificadas da seguinte forma: 0-50 m, 50-140 m, 140-215 m, 215-270 m, 270-320 m, 320-390 m e maiores que $390 \mathrm{~m}$ (Figura 2A). As classes utilizadas foram arbitradas com base na análise da distribuição da altimetria na região da bacia de estudo. 


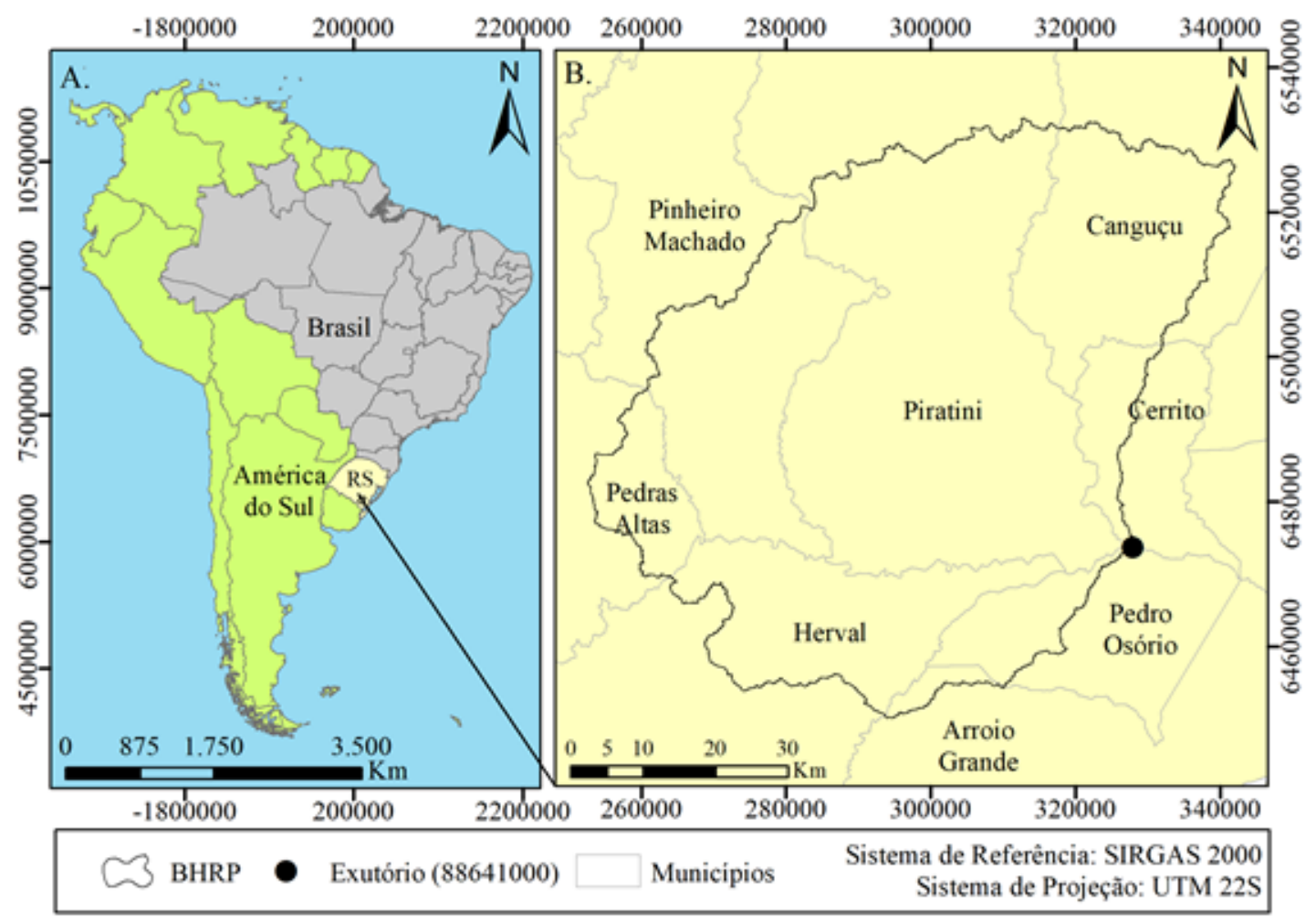

Figura 1. Localização da bacia hidrográfica do rio Piratini (BHRP) (A) e municípios compreendidos pela mesma (B).

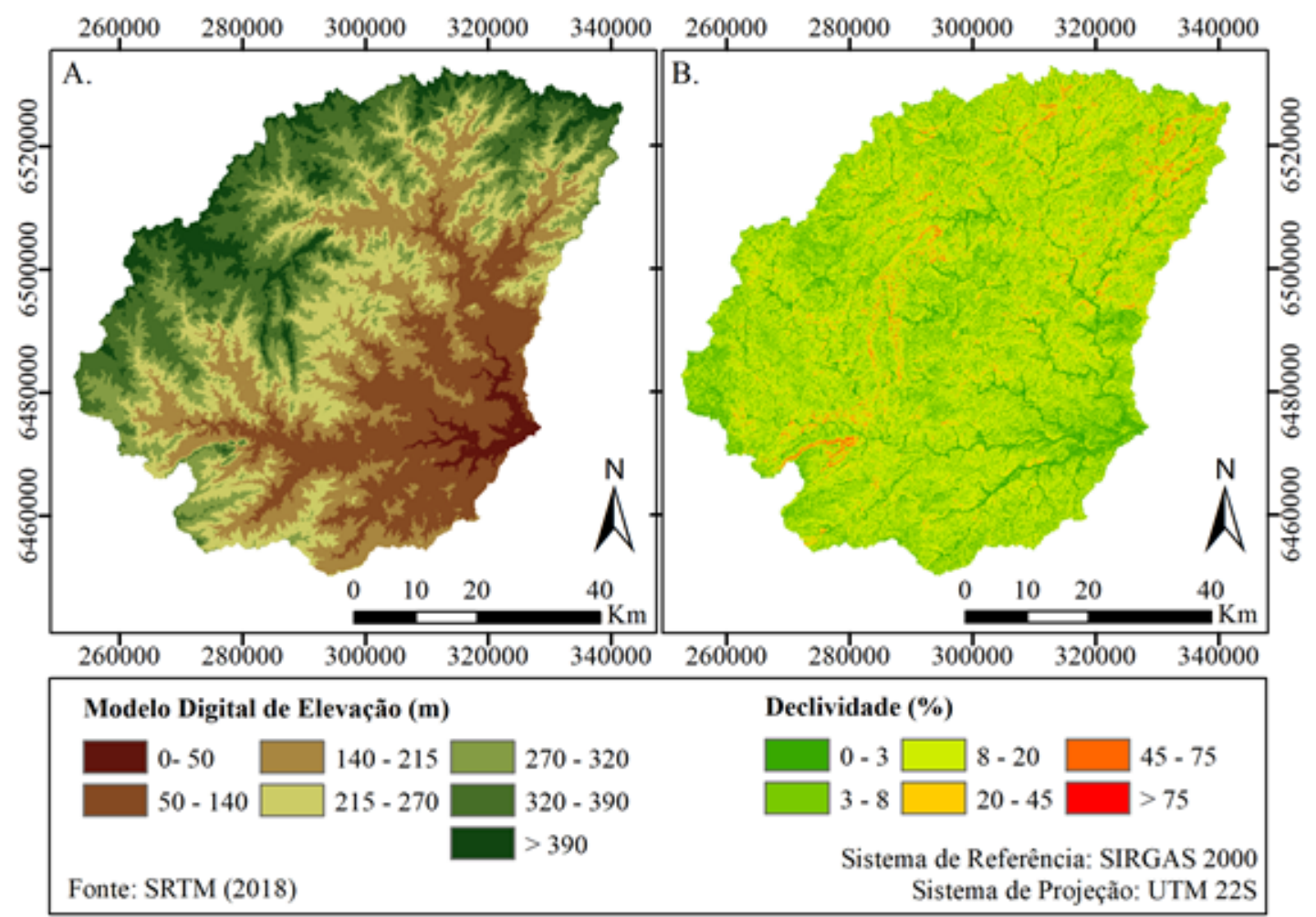

Figura 2. Modelo digital de elevação (A) e mapa de declividades (B) para a bacia hidrográfica do rio Piratini (BHRP)

Para a obtenção do mapa de declividades, em plano $(0-3 \%)$, suave - ondulado $(3-8 \%)$, ondulado porcentagem, foi utilizada a ferramenta Slope do $(8-20 \%)$, forte-ondulado $(20-45 \%)$, montanhoso software ArcGIS ${ }^{\oplus}$. Em seguida, as declividades obtidas $\quad(45-75 \%)$ e forte-montanhoso ( $75 \%$ ) (Figura 2B). foram classificadas de acordo com o proposto pela $\mathrm{O}$ mapa de classes de solo foi obtido junto à EMBRAPA, apresentado em Santos et al. (2014): EMBRAPA, o qual é resultado dos estudos realizados 
por Cunha, Silveira e Severa (2006), na escala 1:50.000. de Argissolos, que ocupam cerca de 66\% da área da As classes pertencentes à BHRP são apresentadas na bacia.

Figura 3, na qual é possível observar a predominância

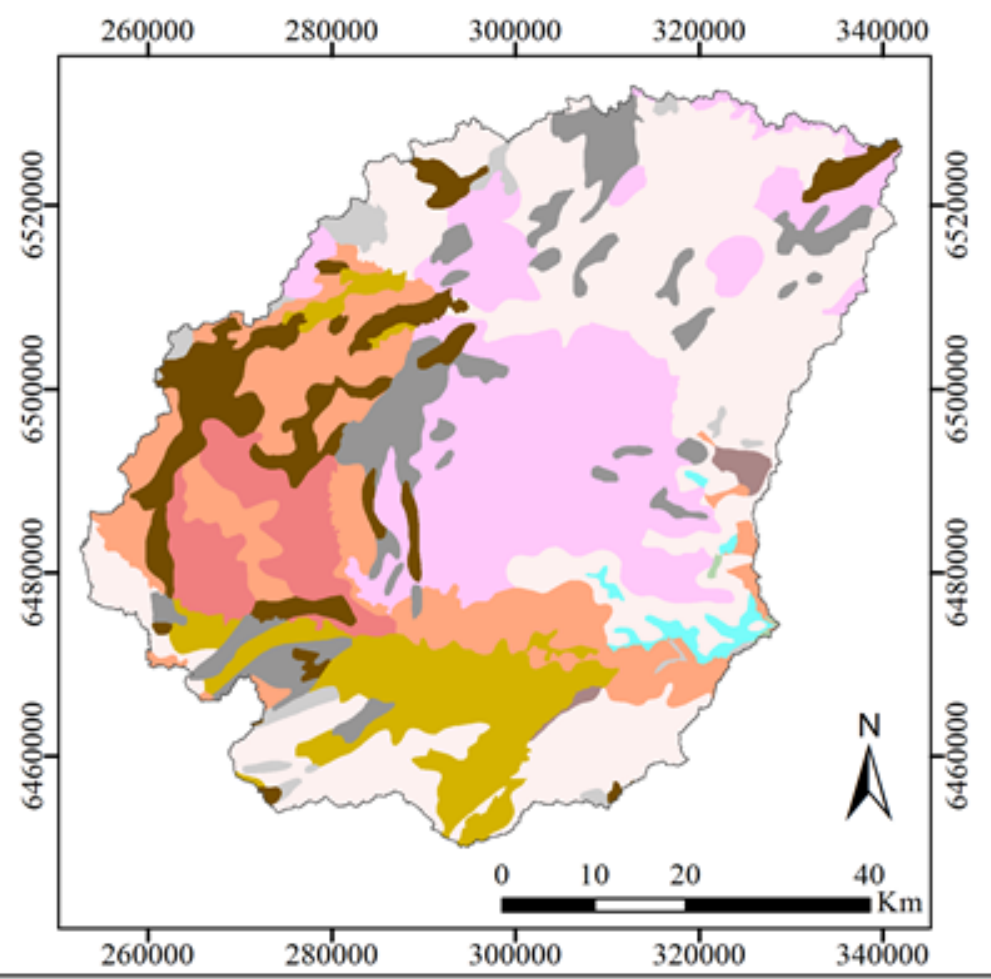

\begin{tabular}{|l|l|l|}
\hline \multicolumn{2}{|c|}{ Classes de solo } & \\
\hline Afloramento rochoso & Argissolo Vermelho-Amarelo & Neossolo Litólico \\
Argissolo Amarelo & Chernossolo Ebânico & Neossolo Regolitico \\
Argissolo Bruno-Acinzentado & Gleissolo Melânico & Planossolo Háplico \\
Argissolo Vermelho & Luvissolo Háplico & \\
Fonte: Cunha, Silveira e Severo (2006) & & Sistema de Referência: SIRGAS 2000 \\
& & Sistema de Projeção: UTM 22S \\
\hline
\end{tabular}

Figura 3. Localização das classes de solo características da bacia hidrográfica do rio Piratini (BHRP)

Para a classificação do uso do solo, foram utilizadas duas imagens Landsat 8, com resoluçáo espacial de $30 \mathrm{~m}$, adquiridas junto ao banco de dados do USGS, referentes às datas 08/02/2018 e 22/10/2018. As imagens foram processadas em composição falsa-cor RGB 654, e classificadas de formasupervisionada através do método da Máxima Verossimilhança (RICHARDS; JIA, 2006). As classes identificadas foram: água, mata, área urbana, solo exposto e agricultura/pastagem, cujas porcentagens representativas da área de estudo estáo apresentadas na Figura 4.

Na Figura 4A, a classe de uso predominante é o solo exposto (41,8\%), seguido de agricultura e pastagem $(31,6 \%)$, enquanto que, na Figura $4 \mathrm{~B}$, esses valores sofrem grandes alteraçóes, com predominância de agricultura e pastagem (65\%) e pouquíssimas áreas com solo exposto (3\%). Essas diferenças já eram esperadas devido ao fato de as imagens utilizadas corresponderem a um período com parte do solo descoberto e um com cobertura vegetal, respectivamente. As classes de mata, área urbana e água tiveram pouca alteração de uma imagem para outra, correspondendo, em média, a 30\% da área da bacia. É importante ressaltar que, durante o processo de classificação, ocorreram algumas confusóes por parte do algoritmo utilizado entre as classes área urbana e solo exposto, principalmente na imagem do dia 08/02, o que implicou em diferenças nos valores de classe urbana entre os períodos. 


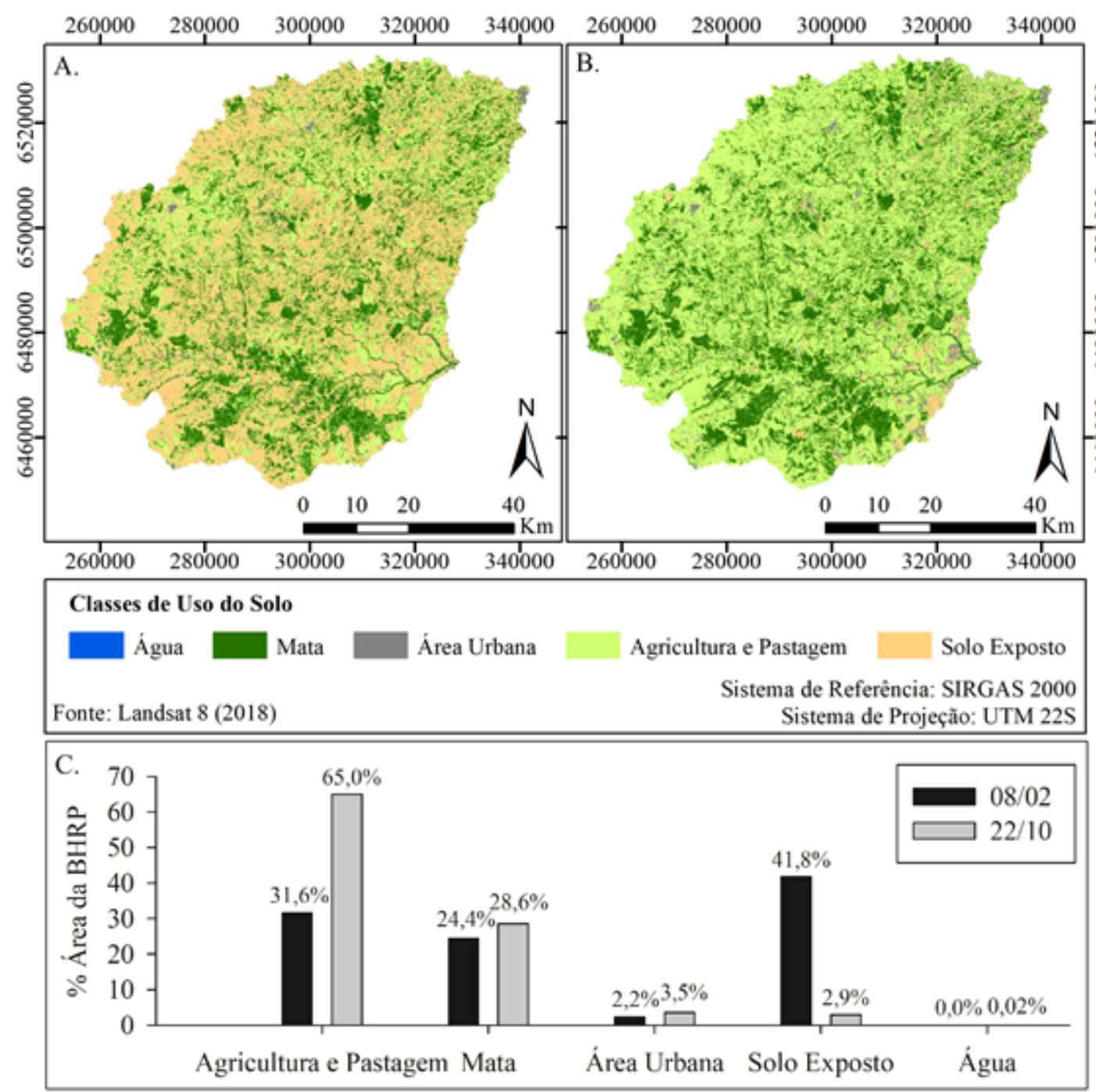

Figura 4. Classes de uso do solo em (A) 08/02/2018, com parte do solo descoberto, e (B) 22/10/2018, com cobertura vegetal; e porcentagem de área das classes para a bacia hidrográfica do rio Piratini (BHRP), em ambos os períodos analisados (C).

\section{Método Analytic Hierarchy Process}

Para obtenção da equação de suscetibilidade à inundação da BHRP, foi utilizado o método Analytic Hierarchy Process (AHP), proposto por Saaty (1977), que combina informações de vários critérios para formar um único índice de avaliação (YU; CHEN; WU, 2011). Este método utiliza um modelo hierárquico para obtenção dos pesos de cada um dos atributos analisados em relação ao objetivo do estudo. Neste estudo, os atributos analisados foram: declividade, altitude, classe de solo e uso do solo.

Para a obtenção do índice de avaliação, duas etapas foram realizadas: i) cálculo do peso associado a cada atributo analisado, a partir de uma matriz de nível hierárquico, e ii) atribuição da nota de 1 a 10 às classes observadas, em cada um dos atributos analisados, conforme maior ou menor suscetibilidade à inundaçáo.
Os atributos selecionados foram organizados numa matriz, na qual foram comparados entre si a partir de fatores que elencam a importância que um exerce sobre o outro. De acordo com Saaty (1977), os valores a serem utilizados na matriz de nível hierárquico, em relação à importância mútua, são: 1/9 (extremamente menos importante), 1/7 (muito menos importante), 1/5 (fortemente menos importante), 1/3 (moderadamente menos importante), 1 (igualmente importante), 3 (moderadamente mais importante), 5 (fortemente mais importante), 7 (muito mais importante) e 9 (extremamente mais importante).

Visando à uniformização dos dados da matriz, foi realizada a divisão dos dados de cada coluna pela soma dos valores da mesma. Posteriormente, a partir da média dos valores resultantes em cada linha, obteve-se os pesos de cada um dos atributos selecionados, cuja 
soma foi igual à unidade. Assim, pôde-se obter uma equação geral para a espacialização da suscetibilidade à inundação, matematicamente descrita pela Eq. 1.

SI $=\mathrm{P}_{\mathrm{D}} \cdot$ Declividade $+\mathrm{P}_{\mathrm{S}} \cdot$ Solo $+\mathrm{P}_{\mathrm{U}} \cdot$ Uso do solo + $\mathrm{P}_{\mathrm{A}} \cdot$ Altitude

Em que, SI é a suscetibilidade à inundação, e $\mathrm{P}_{\mathrm{D}}$, $\mathrm{P}_{S}, \mathrm{P}_{\mathrm{U}}$ e $\mathrm{P}_{\mathrm{A}}$ são os pesos da declividade, do solo, do uso do solo e da altitude, respectivamente.

\section{RESULTADOS E DISCUSSÃO}

As notas adotadas para as classes do relevo foram: $10(0-50 \mathrm{~m}), 8(50-140 \mathrm{~m}), 7(140-215 \mathrm{~m}), 6$ $(215-270 \mathrm{~m}), 3(270-320 \mathrm{~m}), 2(320-390 \mathrm{~m})$ e 1 (maior que $390 \mathrm{~m}$ ); e para a declividade foram: 10 $(0 \%-3 \%), 9(3 \%-8 \%), 6(8 \%-20 \%), 5(20 \%-$ $45 \%), 2(45 \%-75 \%)$ e 1 (maior que $75 \%)$. As notas foram atribuídas de forma que, as regiōes de menores altitudes e/ou mais planas recebessem nota $10 \mathrm{e}$, as com maiores altitudes e/ou mais íngremes recebessem as notas mais baixas, entre 3 e 1 .

As classes de solo receberam notas com base na capacidade de drenagem e profundidade do perfil, tomando como referência a descrição de solos apresentada por Santos et al. (2014). Assim, foi adotada nota 10 para Afloramentos rochosos, 9 para Neossolos,
8 para Gleissolos, 7 para Planossolos e Luvissolos, 6 para Argissolos bruno-acinzentados, 5 para Argissolos amarelos, 4 para Chernossolos e 3 para Argissolos vermelhos e vermelhos-amarelo.

Andrade (2014) afirma que a hierarquização das variáveis analisadas neste processo, oferece a importância das mesmas para a análise fisiográfica do meio quanto ao risco de inundaçáo. Durante a classificação das imagens, vários pixels da classe de água foram classificados como área urbana. Observouse que isso só ocorreu nos trechos próximos à área da bacia, visto que esses pixels eram referentes a açudes e pequenos trechos de hidrografia visíveis entre as matas ciliares. Assim, optou-se pela atribuição de um mesmo peso para estas classes, igual a 8. Para a classe de solo exposto foi atribuída nota 9 , sendo essa considerada a pior condição de uso dentre as classes de uso da BHRP. Esta análise se deu com base na propensão que o solo descoberto tem em facilitar o escoamento superficial direto durante eventos de precipitação.

A classe referente ao uso com agricultura e pastagens recebeu nota 6 e, a mata recebeu nota 2. Para estas classes, teve-se como critério a capacidade da classe de uso em proporcionar infiltração de água no solo e retardo do escoamento superficial direto, além da densidade foliar para interceptação da água.

Tabela 1 - Matriz de nível hierárquico definida para a BHRP e o peso final das variáveis utilizadas para análise da suscetibilidade à inundação

\begin{tabular}{llllll}
\hline & Declividade & Classe de Solo & Uso do Solo & Altitude & Peso \\
\hline Declividade & 1,00 & 3,00 & 3,00 & 7,00 & 0,549 \\
Classe de Solo & $1 / 3$ & 1,00 & 1,00 & 5,00 & 0,225 \\
Uso do Solo & $1 / 5$ & 1,00 & 1,00 & 3,00 & 0,173 \\
Altitude & $1 / 7$ & $1 / 5$ & $1 / 7$ & 1,00 & 0,053 \\
\hline Soma & 1,68 & 5,20 & 5,14 & 16,00 & 1,000 \\
\hline
\end{tabular}

De posse das notas estipuladas e dos pesos arbitrados para cada classe pela metodologia AHP (SAATY, 1977), obteve-se a equação geral para a suscetibilidade à inundação da BHRP (Eq. 2).

SI $=0,549 \cdot$ Declividade+0,225.Solo+0,173. Uso do solo+0,053.Altitude

A suscetibilidade na BHRP variou de 2,172 a 9,721, nos dois cenários propostos. Após a análise de distribuição dos valores de suscetibilidade obtidos, os pixels foram agrupados em classes, definidas com base nos valores com maiores frequências no mapa de SI. As 5 classes definidas, com relação a suscetibilidade à inundação, foram: muito baixa $(<5,0)$, baixa $(5,0$ $-5,8)$, média $(5,8-6,8)$, alta $(6,8-8,2)$ e muito alta $(>8,2)$. A Figura 5 apresenta a espacialização da suscetibilidade à inundação na BHRP, para o período com solo descoberto e para o período com cobertura vegetal. 


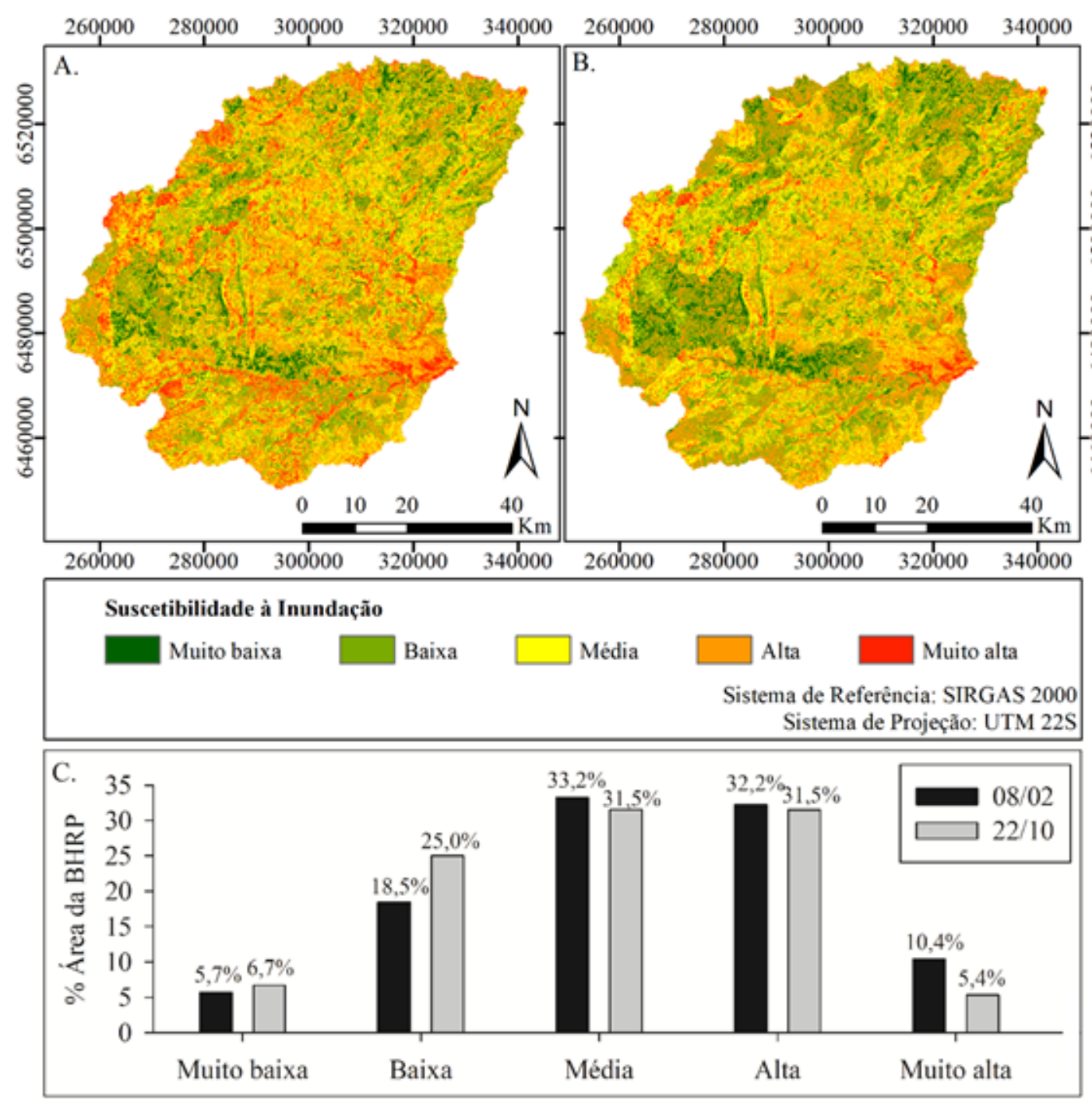

Figura 5. Mapa das classes de suscetibilidade à inundação da bacia hidrográfica do rio Piratini (BHRP) em (A) 08/02/2018, com parte do solo descoberto, e (B) 22/10/2018, com cobertura vegetal; e porcentagem de área das classes de suscetibilidade para a BHRP em ambos os períodos analisados (C).

A partir da Figura 5, é possível perceber que a porcentagem de área das classes "Média" e "Alta" pouco se altera para ambos os períodos analisados. Contudo, percebe-se que a classe "Muito alta" dobra o valor em área quando o solo se encontra exposto, enquanto que, as classes "Muito baixa" e "Baixa" diminuem.

Além disso, os resultados evidenciam a importância da cobertura vegetal para a região, principalmente em áreas agrícolas. $\mathrm{Na}$ bacia, quando a vegetação se encontra estabelecida, estas áreas atuam como medidas de controle, propiciando a infiltração de água no solo e atenuando o pico e o volume de escoamento que chegam nas partes mais baixas, onde se encontram os aglomerados urbanos, que transitaram entre as classes "Alta" e "Muito alta" entre os cenários analisados.

Os resultados obtidos nesse artigo podem ser utilizados também para confirmar os resultados obtidos por Perini et al. (2013), pois o exutório da bacia hidrográfica do rio Piratini fica localizado no centro do município de Pedro Osório, região a qual apresenta de média a muito alta suscetibilidade a inundação, nos dois períodos analisados.

\section{CONCLUSÕES}

Com os resultados obtidos é possível concluir que:

- A suscetibilidade na BHRP teve variaçáo similar nos dois cenários propostos, mas as áreas com baixa e muito baixa suscetibilidade são mais abundantes durante o período com cobertura vegetal, do que no período com menor área de pastagem e agricultura.

- Mesmo em períodos com maior área de solo exposto, há consideráveis áreas da BHRP com baixa ou muito baixa suscetibilidade à inundação.

- As áreas próximas às zonas urbanas apresentam 
suscetibilidade alta ou muito alta durante todo o ano.

- Os resultados obtidos demonstram que a metodologia é eficaz para este tipo de análise, mas recomenda-se a continuidade e aprofundamento da pesquisa, levando em consideração mais fatores e maior intervalo de tempo, tendo em vista que se trata de uma regiâo com histórico de inundaçóes.

\section{AGRADECIMENTOS}

O presente trabalho foi realizado com apoio da Coordenação de Aperfeiçoamento de Pessoal de Nível Superior - Brasil (CAPES) e da Fundação de Amparo à Pesquisa do Estado do Rio Grande do Sul (FAPERGS).

\section{LITERATURA CITADA}

AK1NC1, H.; ÖZALP, A. Y.; TURGUT, B. Agricultural land use suitability analysis using GIS and AHP technique. Computers and Electronics in Agriculture, v. 97, p. 71-82, 2013.

ALVARES, C. A.; STAPE, J. L.; SENTELHAS, P. C.; GONÇALVES, J. L. M.; SPAROVEK, G. Koppen's climate classification map for Brazil. Meteorologische Zeitschrift, v. 22, n. 6, p. 711-728, 2013.

ANDRADE, S. L.; FERREIRA, V. O.; SILVA, M. M. Elaboração de um mapa de risco de inundaçôes da bacia hidrográfica do córrego São Pedro, área urbana de Uberlândia-MG. Caderno de Geografia, v.24, n.41, p. 1-16, 2014.

CUNHA, N.G.; SILVEIRA, R.J.C.; SEVERO, C.R.S.

Solos e terras do planalto Sul-Rio-Grandense e planícies costeiras. Pelotas: Embrapa Clima Temperado, 2006. 42 p. (Embrapa Clima Temperado. Circular técnica, 55).

ESRI, ArcGIS DESKTOP 10.1. Redlands, CA: Environmental Systems Research Institute, Inc. (Esri), 2014. CD-ROM.

FEIZIZADEH, B.; BLASCHKE, T. GIS-multicriteria decision analysis for landslide susceptibility mapping: comparing three methods for the Urmia lake basin, Iran. Natural Hazards, v. 65, p. 2105-2128, 2013.
GOUVÊA, T.; ZARNOT, D. H.; ALBA, J. M. F. Caracterização geoambiental e histórico do processo de desenvolvimento da bacia da Lagoa Mirim. In: ALBA, J. M. F. Sustentabilidade Socioambiental da Bacia da Lagoa Mirim. Pelotas: Embrapa Clima Temperado, 2010. Cap. 1, p. 19-30.

LEITE, M. E.; ROCHA, A. M. Mapeamento da susceptibilidade à inundação em áreas urbanas: o caso da cidade de Montes Claros-MG. Geosul, v. 31, n. 62, p 125-150, 2016.

LIM, J.; LEE, K. Investigating flood susceptible areas in inaccessible regions using remote sensing and geographic information systems. Environmental Monitoring and Assessment, v. 189, n. 3, p. 96, 2017.

MAGALHÁES, I. A. L.; THIAGO, C. R. L.; AGRIZZI, D. V.; SANTOS, A. R. Uso de geotecnologias para mapeamento de áreas de risco de inundação em Guaçuí, ES: uma análise comparativa entre dois métodos. Cadernos de Geociências, v. 8, n. 2, p. 63-70, 2011.

MARCHEZETTI, A. L.; KAVISKI, E.; BRAGA, M. C. B. Aplicação do método AHP para a hierarquização das alternativas de tratamento de resíduos sólidos domiciliares. Ambiente Construído, v. 11, n. 2, p. 173-187, 2011.

MCKENZIE, A. A.; RUTTER, H. K.; HULBERT, A. G. The use of elevation models to predict areas at risk of groundwater flooding. Geological Society, London, Special Publications, v. 345, n. 1, p. 75-79, 2010 .

OLIVEIRA, G. G.; FLORES, T.; BRESOLIN JUNIOR, N. A.; HAETINGER, C.; ECKHARDT, R. R.; QUEVEDO, R. P. Análise da suscetibilidade a inundaçôes e enxurradas na bacia hidrográfica do Rio Forqueta, RS, Brasil. Geociências, v. 37, n. 2, p. 437 453, 2019. 
PERINI, A. B.; MARCUZZO, F. F. N.; SILVA, D. R. A. Modelos Digitais de Elevação no mapeamento de suscetibilidade de inundação no município de Pedro Osório/RS. In: XVI Simpósio Brasileiro de Sensoriamento Remoto - SBSR, Anais..., Foz do Iguaçu, PR, Brasil, 13 a 18 de abril de 2013, INPE, p. 6417-6424.

RICHARDS, J. A.; JIA, X. Remote sensing digital image analysis: an introduction. New York: Springer Verlag, 2006. 494 p.

SAATY, T. L. A scaling method for priorities in hierarchical structures. Journal of Mathematical Psychology, v. 15, n. 3, p. 234-281, 1977.

SAAVEDRA, O. F. C.; MUÑOZ, V. A.; REIS, J. B. C. Delimitação da planície inundável e avaliação a partir de um evento de inundação ocorrido no município de São Borja (RS). In: XVII Simpósio Brasileiro de Sensoriamento Remoto - SBSR, Anais..., João Pessoa, PB, Brasil, 25 a 29 de abril de 2015, INPE, p. 62906297.

SANTOS, H. G.; JACOMINE, P. K. T.; ANJOS, L. H. C.; OLIVEIRA, V. A.; LUMBRERAS, J. F.; COELHO, M. R.; ALMEIDA, J. A.; CUNHA, T. J. F; OLIVEIRA, J. B. Sistema Brasileiro de Classificaçáo de Solos. 4. ed. Brasília: Embrapa, 2014. 376p. ISBN 978-85-7035-359-7

SHAHABI, H.; KHEZRI, S.; AHMAD, B. B.; HASHIM, M. Landslide susceptibility mapping at central Zab basin, Iran: A comparison between analytical hierarchy process, frequency ratio and logistic regression models. Catena, v. 115, p. 55-70, 2014.

SAHARIA, M.; KIRSTETTER, P.; VERGARA, H.; GOURLEY, J. J.; HONG, Y.; GIROUD, M. Mapping Flash Flood Severity in the United States. Journal of Hydrometeorology, v. 18, p. 397-411, 2017.

VIECELI, N.; BORTOLIN, T. A.; MENDES, L. A.; BACARIM, G.; CEMIN, G.; SCHNEIDER, V. E.
Morphometric evaluation of watersheds in Caxias do Sul City, Brazil, using SRTM (DEM) data and GIS. Environmental Earth Sciences, v. 73, p. 5677-5685, 2015.

YALCIN, A.; S. REIS, S.; A.C. AYDINOGLU, A. C.; YOMRALIOGLU, T. A GIS-based comparative study of frequency ratio, analytical hierarchy process, bivariate statistics and logistics regression methods for landslide susceptibility mapping in Trabzon, NE Turkey. Catena, v. 85, p. 274-287, 2011.

YOUSSEF, A. M.; PRADHAN, B.; HASSAN, A. M. Flash flood risk estimation along the St. Katherine road, southern Sinai, Egypt using GIS based morphometry and satellite imagery. Environmental Earth Sciences, v. 62, p. 611-623, 2011.

YU, J.; CHEN, Y.; WU, J. Cellular automata based spatial multi-criteria land suitability simulation for irrigated agriculture. International Journal of Geographical Information Science, v. 25, n. 1, p. 131-148, 2011.

ZHANG, F.; ZHU, X.; LIU, D. Blending MODIS and Landsat images for urban flood mapping. International Journal of Remote Sensing, v. 35, n. 9, p. 3237-3253, 2014. 